Mediscope

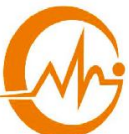

The Journal of GMC

\title{
Antibiotic resistance: a crisis needs to be encountered
}

\author{
S Saha ${ }^{1 凶}$, KS Ahmed $^{2}$, S Islam ${ }^{3}$, MM Islam $^{4}$, MS Khan $^{5}$, C Saha ${ }^{6}$
}

\begin{abstract}
Bacteria are said to be resistant if their growth is not halted by the maximum level of an antibiotic that is tolerated by the host. Antimicrobial agents are among the most frequently prescribed and inappropriate use of these agents are associated with allergic reactions, toxicities, super infection and more importantly the development of antimicrobial resistance. Antibiotics are frequently prescribed inappropriately in terms of type, dose, duration and indication. The excessive and inappropriate use of antibiotics add unnecessary economic burden to the healthcare system and cause increasing antibiotic resistant organisms which result use more of expensive and toxic drugs. It is known that patients infected with drug-resistant organisms are more likely to require hospitalization, have a longer hospital stay and death. And all efforts to contain the problem must include manufacturers, prescribers, dispensers and consumers of antimicrobial agents to work must work in cooperation for prevention of antibiotic resistance.
\end{abstract}

Key words: antibiotic resistance, rational use of antibiotic.

\section{Background}

Antibiotic resistance is a worldwide problem. It can cross international boundaries and spread between continents with ease. Many forms of resistance spread with remarkable speed. World health leaders have described antibiotic resistant microorganisms as "nightmare bacteria" that "pose a catastrophic threat" to people in every country in the world. ${ }^{1}$ We are enjoying the benefits of some wonder drugs from their discovery. And those wonder drugs are nothing but antibiotics. For the last 60-65 years since the antibiotic penicillin was first used, bacterial assaults on us have been controlled, diminished, and cured.
The great power of antibiotics, however, is failing. This failure is due to what has been called an international public health nightmare that is increasing bacterial resistance to many antibiotics that once cured bacterial diseases readily. ${ }^{2}$ In other words, the antibiotic is unable to destroy the bacteria. Since antibiotics were first introduced, it was noticed that some bacteria would not respond to them. Bacterial resistance has always been around. Resistant genes preexisted in nature, in soil, and water, and their presence was probably related to the production of antibacterial agents, synthesized naturally in the environment. ${ }^{3}$ The excessive and inappropriate use of antibiot-

1. Assistant Professor \& Head, Department of Pharmacology \& Therapeutics, Ad-din Sakina Medical College, Jessore and PhD fellow, Bangladesh University of Professionals, Dhaka. Email: dr.sanjoysahammc@gmail.com

2. Professor (cc) \& Head, Department of Forensic Medicine, Ad-din Sakina Medical College, Jessore

3. Associate Professor \& Head, Department of Community Medicine, Ad-din Sakina Medical College, Jessore

4. Assistant Professor \& Head, Department of Pharmacology \& Therapeutics, Ad-din Sakina Medical College, Jessore

5. Assistant Professor, Department of Forensic Medicine, Ad-din Sakina Medical College, Jessore

6. Lecturer, Department of Pharmacology \& Therapeutics, Ad-din Sakina Medical College, Jessore 
ics adds in an unnecessary economic burden to healthcare system and coincides with an increase in drug resistant organisms, which has resulted in the use of more expensive and toxic drugs. It is known that patients infected with drug-resistant organisms are more likely to require hospitalization, have a longer hospital stay and prone to die. ${ }^{4}$

In Bangladesh, misuse and waste of antibiotics appear to be frequent. Over the-counter availability of all types of antibiotics makes the situation worse. The widespread and inappropriate use of antibiotic results in the development of a progressively antibioticresistant microbial ecosystem in Bangladesh. This is clearly indicated by the high prevalence of antibiotic resistance among community-acquired Shigella, Salmonella, Vibrio cholerae, Escherichia coli, Neisseria gonorrhoe, Mycobacterium tuberculosis, Sptreptococcus pneumoniae and Haemophilus influenzae infections in Bangladesh. ${ }^{4}$

Penicillin was first discovered in $1928 .^{5}$ In 1969, the US Surgeon General summarized this enthusiasm with the following historical words to the Congress "The time has come to close the book on infectious disease." While many praised this vision, the realities of infectious diseases were to take an unexpected and completely different course in the following period. Even before penicillin was used clinically, Abraham and colleagues had discovered an enzyme capable of destroying penicillin. ${ }^{6,7}$ By 1950, half of the $S$. aureus isolates were resistant to penicillin.8 Penicillin-resistance first became prevalent among hospital-acquired staphylococci, but by the late 1960 s also in community acquired infections. ${ }^{9,10}$ However, the implications of antimicrobial resistance were seriously underestimated and there was widespread confidence that science would find new solutions to this problem.

Methicillin, introduced in 1959, offered a solution for treating penicillin-resistant staphylococci, however, already in 1961 Jevons described the first methicillinresistant $S$. aureus (MRSA). ${ }^{11}$ Vancomycin was approved for clinical use in 1958 and was suitable to treat MRSA, and unfortunately by 1986 , vancomycin-resistance started to emerge in enterococci in Europe. ${ }^{12}$ The infectious disease experts feared that the much more virulent $S$. aureus would acquire resistance to vancomycin too. In 1997, vancomycin-intermediate resistant $S$. aureus (VISA) was discovered in Japan. ${ }^{13}$ In 1948, Guiseppe Brotzu discovered that a substance produced by cephalosporium acremonium effectively killed Salmonella typhi, laying the foundation for a whole new group of $\beta$-lactam antibiotics, the cephalosporins. ${ }^{14}$ Starting with the use of cefalotin in 1964, the first-generation cephalosporins were succeeded by secondgeneration cephalosporins such as cefuroxime, and later on the third-generation oximino-cephalosporins, such as cefotaxime and ceftriaxone, which became fundamental in the treatment of gram-negative bacteria, and ceftazidime, which had additional antipseudomonas effect.

Ampicillin, the first penicillin with a broadspectrum and activity against gram-negative bacteria, was introduced in the early 1960s. Shortly after, Datta and colleagues in Greece described in a strain of $E$. coli a plasmidmediated ampicillin-hydrolyzing $\beta$-lactamases, which was named TEM-1 after the patient, whose name was Temoniera. ${ }^{15}$ In the coming years, mutations have lead to the emergence of a large number of extendedspectrum $\beta$-lactamases enzymes. ${ }^{16}$ The optimism of the "golden age" of antibiotics, has given way to a reserved feeling, as bacteria have generated resistance against virtually any antimicrobial agent that humans have developed. While the pharmaceutical industry has largely been passive in developing new antimicrobials the last few decades seem to herald that we may be entering what Cohen called the post-antibiotic area. ${ }^{17}$

\section{Types and causes}

The types of resistance are primary resistance when some bacteria posses an innate property to certain drug, and acquired resistance when either from mutation or gene transfer. The causes of antimicrobial resistance are a) indiscriminate use of antimicro- 
bial resistance are a) indiscriminate use of antimicrobials, b) use of wrong antimicrobials, c) use of right antimicrobials, but not given in right doses, d) interval \& duration, e) use of less quality antimicrobials, and f) overuse or abuse of antibiotics in non-humans.

\section{Brief mechanism of development of resis- tance}

Genetic alterations leading to drug resistance are developed by naturally resistant strains, spontaneous mutation, and transmission of genes from other organisms by plasmids (extra-chromosomal genetic elements) and bacteriophages (virus infected bacteria). Altered expression of proteins is occurred in drug resistant organism such as bacteria produce enzyme that inactivate drug, bacteria synthesize modified target against which drug has no effect, bacteria reduce permeability of a drug, bacteria actively export drugs using "multidrug resistance pump" (MDR pump or efflux pump), etc.

\section{Common antibiotic resistant pathogens in Bangladesh}

In Bangladesh, misuse and waste of antibiotics appear to be frequent. Over the-counter availability of all types of antibiotics makes the situation worse. Antibiotic prescribing by the physicians appears to be less than ideal. The widespread and inappropriate use of antibiotic results in the development of a progressively antibiotic-resistant microbial ecosystem in Bangladesh. This is clearly indicated by the high prevalence of antibiotic resistance among community-acquired Shigella, Salmonella, Vibrio cholerae, Escherichia coli, Neisseria gonorrhoe, Mycobacterium tuberculosis, Sptreptococcus pneumoniae and Haemophilus influenzae infections in Bangladesh. Antibiotic resistance is also frequent in human gut flora in Bangladesh. A high prevalence of resistant gut flora in healthy human and probably in animals appears to be the source of antimicrobial resistance genes, the dissemination of which is enhanced by extensive use and misuse of antimicrobial pathogens agents and by environmental conditions such as crowding, poor sanitation or contamination of food.
Emergence and spread of antibiotic resistance

There are many factors that contribute to the emergence, persistence and spread of resistant bacteria. Selective pressure on microorganisms resulting from imprudent indiscriminate use of antibiotics in humans and animals is the key factor for the emergence of antibiotic resistance gene among bacterial population. ${ }^{4}$ Mobile genetic elements such as plasmid or transposon arries transports antibiotic resistance gene from one bacterium to another bacterium of the same or different species or genus. ${ }^{4}$ The intensive use of antibiotics, for treatment and prophylaxis, make hospital a prime site for the emergence, maintenance and spread of resistant pathogens. After discharge of patients from the hospital, resistant pathogens establish in the community and are transmitted from persons to persons or persons to household pets or vice versa. ${ }^{4,18}$ More than half of the total production of antimicrobials in the world is used in farm animals and in agriculture. All of these uses contribute significantly to the emergence, maintenance and spread of resistant pathogens in the community.

\section{Detection of antibiotic resistance}

Antibiotic resistance in bacteria is commonly detected as a part of standard clinical microbiological procedure for the identification of cause of infection and appropriate treatment. Detection depends on the collection of clinical samples from the patient, and on the isolation, identification and susceptibility testing of the pathogens in good clinical microbiology laboratory by trained personnel in a cost- effective way. A good representative database on the current status of antibiotic resistance among common and important pathogens is essential for the proper treatment of infectious diseases in the country. ${ }^{4}$

Measures to be encountered against antimicrobial resistance

Energetic measures to slow down the emergence and spread of antimicrobial resistance should include programmes on surveillance, 
education and research on antimicrobial resistance, and regulation of use of antimicrobials in the hospitals and in the community. The efforts include- a) detection and awareness of problem of antimicrobial resistance by microbiology laboratory and public media, b) surveillance (local, regional and international) on antimicrobial resistance should be established in collaboration with World Health Organization ${ }^{19}$, c) guidelines for antibiotic use and committee should be developed for each country, d) antibiotic use in hospitals should be regulated and rationalized, e) detection and targeting the resistance, f) education on antibiotic resistance to manufacturers, prescribers, dispensers and consumers through continuing education and other means, g) vaccines for preventing infectious diseases should be encouraged in all circumstances, h) hygiene and sanitation should be improved and practiced, and i) nutritional status should be maintained and improved. ${ }^{4}$

\section{Guidelines for using antibiotics}

The following activities should in action such as diagnosis of infections whether community or nosocomial in nature; isolation or prediction of infecting pathogen; knowledge about antibiotic susceptibility of the pathogen in the locality, country and region; consulting clinical microbiologist for opinion, if necessary; selection of an appropriate narrow spectrum, low- cost, $1^{\text {st }}$-choice bactericidal drug; prescription for appropriate duration; an adequate concentration at the site of infection should be ensured; and informing patients about potential side-effects. ${ }^{4}$

\section{Conclusion}

A effective national and international collaboration is urgently needed for prudent and rational use of antimicrobials in human and animals through more stringent regulations of marketing, sale, use and consumption essential for the prevention and control of antimicrobial resistance. And, all efforts to contain the problem, manufacturers, prescribers, dispensers and consumers of antimicrobial agents must work in cooperation for prevention of antibiotic resistance.

\section{References}

1. Roberts RR, Hota B, Ahmad I, et al. Hospital and societal costs of antimicrobialresistant infections in a Chicago teaching hospital: implications for antibiotic stewardship. Clin Infect Dis 2009;49(8):1175-84.

2. Levy SB. The challenge of antibiotic resistance. Sci Am 1998;278(3):46-53.

3. Bergogne-Berezin $\mathrm{E}$. Who or what is the source of antibiotic resistance? J Med Microbiol 1997;46(6);461-4.

4. The ORION 1998;1. Available from http://www.orion-group.net/journals/ Journals/Vol1_Sep1998/start.htm (accessed on December 15, 2017).

5. Fleming $A$. On the antibacterial action of cultures of a penicillium, with special reference to their use in the isolation of $B$. influenzae. $\mathrm{Br} J$ Exp Pathol 1929;10(3):226-36.

6. Abraham EP, Chain E. An enzyme from bacteria able to destroy penicillin. Nature 1940;146:837.

7. Kirby WMM. Extraction of highly potent penicillin inactivator from penicillin resistant staphylococci. Science 1944; 99:452-3.

8. Livermore DM. Antibiotic resistance in staphylococci. Int $\mathrm{J}$ Antimicrob Agents 2000;16(Suppl 1):S3-10.

9. Barber M. Methicillin-resistant staphylococci. J Clin Pathol 1961;14(4):385-93.

10.Jessen O, Rosendal K, Bülow $P$, Faber V, Eriksen KR. Changing staphylococci and staphylococcal infections. A ten-year study of bacteria and cases of bacteremia. N Engl J Med 1969;281(12):627-35.

11.Jevons MP. Celbenin-resistant staphylococci. Br Med J 1961;1:124-5.

12.Levine DP. Vancomycin: a history. Clin Infect Dis 2006;42(Suppl 1):S5-12.

13. Hiramatsu K, Hanaki H, Ino T, Yabuta K, Oguri T, Tenover FC. Methicillin resistant Staphylococcus aureus clinical strain with reduced vancomycin susceptibility. J Antimicrob Chemother 1997;40(1):135-6.

14. The History Of Antibiotics. Available from https://www.herbs2000.com/medica/2_an tibioticsH3.htm (accessed on December 15, 2017).

15.Datta N, Kontomichalou P. Penicillinase synthesis controlled by infectious $R$ 
factors in Enterobacteriaceae. Nature 1965;208(7):239-41.

16.Bradford PA. Extended-spectrum betalactamases in the 21st century: characterization, epidemiology, and detection of this important resistance threat. Clin Microbiol Rev 2001;14(4):933-51.

17.Cohen ML. Epidemiology of drug resistance: implications for a post antimicro- bial era. Science 1992;257(5073):1050-5.

18. Acar JF. Consequences of bacterial resistance to antibiotics in medical practice. Clin Infect Dis 1997;24(Suppl 1):S17-8.

19. World Health Organization. Communicable Diseases Cluster. Overcoming antimicrobial resistance. Geneva: World Health Organization; 2000.

\section{Suggestion for citation of the above:}

Saha S, Ahmed KS, Islam S, Islam MM, Khan MS, Saha C. Antibiotic resistance: a crisis needs to be encountered. Mediscope 2018;5(1):33-7. 